\title{
Antidepressant effect of geranylgeranylacetone in a chronic mild stress model of depression and its possible mechanism
}

\author{
JING-MEI ZHONG $^{1 *}$, SHAO-YUAN WU ${ }^{1 *}$, JIE BAI $^{2}$, QIANG GUO $^{1}$, JIAN TAO $^{1}$, \\ HUI CHEN ${ }^{1}$, NAI-WEI ZHAO ${ }^{1}$, ZHONG ZHAO ${ }^{1}$ and $\mathrm{HAO} \mathrm{FU}^{1}$ \\ ${ }^{1}$ Department of Neurology, The First People's Hospital of Yunnan, Kunhua Affiliated Hospital of Kunming Medical University, \\ Kunming 650032; ${ }^{2}$ Medical Faculty, Kunming University of Science and Technology, Kunming 650500, P.R. China
}

Received May 24, 2012; Accepted August 9, 2012

DOI: $10.3892 /$ etm.2012.669

\begin{abstract}
Depression is a highly debilitating and widely distributed illness in the general population. Geranylgeranylacetone (GGA), a non-toxic anti-ulcer drug, has been reported to have protective effects in the central nervous system. The aim of this study was to determine the antidepressant effect of GGA in a chronic mild stress (CMS) model of depression. We confirmed that CMS in rats caused a reduction in locomotor activity and an increase in the levels of monoamine oxidase-A (MAO-A) and caspase-3 in the hippocampus. GGA treatment reversed stress-induced alterations in locomotor activity and target levels of MAO-A and caspase-3. In addition, GGA treatment induced heat shock protein 70 (Hsp70) expression in the hippocampus. These findings suggest that GGA possesses an antidepressant activity in a CMS model of depression. The activity of GGA in the relief of depression may be mediated via the induction of Hsp70 expression to suppress MAO-A expression and the apoptosis cascade.
\end{abstract}

\section{Introduction}

Depression is a common mental disorder, with main characteristics including regular negative moods, decreased physical activity, loss of interest in usual activities, feeling of helplessness and suicidal tendencies (1). Although depression has been widely studied, the pathogenesis of depression remains unknown. Until now, antidepressants available on the pharmaceutical market mainly include tricyclic antidepressants, monoamine oxidase inhibitors, selective serotonin reuptake inhibitors and serotonin-noradrenergic reuptake inhibitors (2).

Correspondence to: Dr Jing-Mei Zhong or Dr Qiang Guo, Department of Neurology, The First People's Hospital of Yunnan, Kunhua Affiliated Hospital of Kunming Medical University, No. 157 Jinbi Road, Kunming 650032, P.R. China

E-mail: rosezhong92@yahoo.com.cn

E-mail: gqkj003@sina.com

*Contributed equally

Key words: depression, geranylgeranylacetone, heat shock protein 70 , apoptosis, monoamine oxidase
However, numerous antidepressants frequently produce side-effects, including sedation, sleep disturbance, cognitive impairment and sexual dysfunction (3). Accordingly, the development of more effective antidepressants without any (or with fewer) adverse effects is required.

Heat shock protein 70 (Hsp70) functions as a molecular chaperone that mediates a highly conserved system of cellular responses to various stimuli (4). When the cell is exposed to stress, Hsp70 is induced to maintain cellular homeostasis $(5,6)$. Several reports have shown that $\mathrm{Hsp} 70$ has a protective role in various models of nervous system injury, including oxidative stress and ischemic-reperfusion injury (6-8). In recent years, a huge body of evidence has accumulated suggesting that the activity of Hsp70 is associated with the pathogenesis of depression. The activity of the glucocorticoid receptor, which plays an important role in depression, is regulated by Hsp70 $(9,10)$. The 162-base deletion in the 5'-flanking region of Hsp70 gene mRNA was observed in patients with depression (11). It also has been shown that antidepressants may increase Hsp70 expression, which is a possible mechanism underlying the therapeutic efficacy of antidepressants (12-14). As such, Hsp70 is a new therapeutic target for depression.

Geranylgeranylacetone (GGA) is an acyclic isoprenoid compound that has been widely used in clinic as an anti-ulcer drug. Numerous studies have demonstrated that GGA is a non-toxic Hsp70 inducer, which safely induces Hsp70 expression in gastric mucosa, intestine, liver, heart and retina (15-18). GGA is a lipid-soluble reagent and easily crosses the blood-brain barrier to exert neuroprotective activity. It has been reported that GGA administration induced the expression of heat shock proteins including Hsp70 and suppressed polyglutamine toxicity in cell culture and mouse models of spinal and bulbar muscular atrophy (19). GGA is also relevant to the treatment and prevention of various neural diseases, including ischemia-reperfusion injury and morphine addiction (20-22).

However, the effect of GGA on depression has not yet been investigated. In the present study, we suggest that when GGA is administered, it induces Hsp70, which may in turn alleviate behavioral abnormalities in depression. The present study was performed to investigate the possible antidepressant effects of GGA in the chronic mild stress (CMS) model of depression in rats. 


\section{Materials and methods}

Reagents. GGA was purchased from Eisai (Tokyo, Japan). Antibodies [Hsp70, monoamine oxidase-A (MAO-A), caspase-3 and $\beta$-actin] were obtained from Santa Cruz Biotechnology, Inc. (Santa Cruz, CA, USA). DAB color developing reagent was purchased from Boao Seng Company (Beijing, China). TRIzol reagent was obtained from Molecular Research Center Company (Cincinnati, OH, USA). RevertAid $^{\mathrm{TM}}$ First Strand cDNA Synthesis kit and dNTPS were purchased from Fermentas (Walldorf, Baden, Germany).

Animals. Male Sprague-Dawley rats (Chengdu Dashuo Biological Technology Co., Ltd., Chengdu, China) weighing $180 \pm 20 \mathrm{~g}$ were used in the experiments. The rats were allowed to habituate to the housing facilities for 1 week before the experiments began. Rats were maintained on a $12 \mathrm{~h}$ light-dark cycle and had free access to food and water. The use of animals was performed in accordance with the National Institutes of Health Guide for the Care and Use of Laboratory Animals and approved by the Local Committee on Animal Use and Protection.

CMS procedure. The method of stress was modified according to previous studies and previous practice in our laboratory. The stressed groups were subjected to the following stressors for three weeks: water and food deprivation for $24 \mathrm{~h}$; physical restraint for $30 \mathrm{~min}$ (rats were placed into small iron compartments, which were $15-\mathrm{cm}$ high and hand-made; the diameter of this compartment could be adjusted appropriately from $3-6 \mathrm{~cm}$ according to the size of rats); rotation for $30 \mathrm{~min}$ (motor-driven rotator, the velocity of rotation was $33 \mathrm{rpm}$ ); damp environment for $12 \mathrm{~h} \mathrm{(200} \mathrm{ml} \mathrm{water} \mathrm{was} \mathrm{added} \mathrm{to} 100 \mathrm{~g}$ bedding); inclined cage for $12 \mathrm{~h}$ at $45^{\circ}$; the rats were combined with new invader rats for $24 \mathrm{~h}$; daytime reversed and night reversed for $12 \mathrm{~h}$, respectively. The order of each stress was randomly arranged and one type of stress was performed daily. The rats were randomly divided into 3 groups: the control group (control, $n=12$ ); the stress group (stress, $n=12$ ); the stress $+1,000 \mathrm{mg} / \mathrm{kg} \mathrm{GGA}$ group (stress $+\mathrm{GGA}, \mathrm{n}=12$ ). The rats of the stress $+1,000 \mathrm{mg} / \mathrm{kg}$ GGA group were administered with GGA $(1,000 \mathrm{mg} / \mathrm{kg})$. The rats of the stress and control groups were injected intraperitoneally with saline (NS).

Open field test. The open-field test was conducted in a four-sided 100x100x50 cm box, divided into 25 equilateral smaller squares. The test was conducted under a sedate environment. The rats were placed onto the center square of the box, then their activity was measured for $5 \mathrm{~min}$. The number of crossings and rearings were scored manually. The apparatus was cleaned between tests.

Immunohistochemistry analysis. The rats were sacrificed after the behavioral test by deep anesthesia. The brain samples were placed into the $4 \%$ paraformaldehyde for fixation overnight, followed by a wash with a flow of water and dehydration by gradient alcohol. The paraffin samples were sliced to a thickness of $5 \mu \mathrm{m}$. Endogenous peroxidase was blocked with blocking solution $\left(3 \% \mathrm{H}_{2} \mathrm{O}_{2}\right.$ in methanol) for $15 \mathrm{~min}$, following PBS washing. The slices were incubated in rabbit anti-mouse polyclonal Hsp70 (1:100 dilution) for $1 \mathrm{~h}$ at room temperature, and then incubated with biotinylated goat anti-rabbit $\operatorname{IgG}$ for $1 \mathrm{~h}$. After rinsing with PBS, the slices were incubated with albumin fluid labeled with horseradish peroxidase for $1 \mathrm{~h}$ and then DAB solution was utilized for coloration. The slices were counterstained with hematoxylin.

Reverse transcription-polymerase chain reaction (RT-PCR). The rats were sacrificed after the behavioral test by deep anesthesia. The right hippocampus of rats was rapidly dissected out, frozen and stored in a deep freezer at $-80^{\circ} \mathrm{C}$ until the assays. The total RNA of the hippocampus was isolated using TRIzol reagent following the manufacturer's instructions. The RNA level was measured by an ultraviolet spectrophotometer by OD 260 measurements. cDNA was synthesized by reverse transcription. PCR amplification was performed with primers designed for the genes of interest. The primers used were as follows: HSP-70 sense, 5'-GCTGGTGAGCCACTTCGTG-3' and antisense, 5'-TGGATCTGCGCCTTGTCC-3' (Hsp70 PCR production, 288bp);MAO-A sense,5'-ATTGGAGGCGGCATC TCAGGAT-3' and antisense, 5'-AGGTGGGAATGCACC ACGGAAT-3' (MAO PCR production, 288 bp); caspase-3 sense, 5'-AACGAACGGACCTGTGG-3' and antisense, 5'-TTT GCATGGAAA GTGGC-3' (caspase-3 PCR production, $\quad 390 \quad$ b p ); $\quad \beta-$ a ctin sense, 5'-CACTGCCGCATCCTCTTCCTC-3' and antisense, 5'-CTCCTGCTTGCTGATCCACAT-3' ( $\beta$-actin PCR production, $400 \mathrm{bp}$ ). The PCR products were separated by $2 \%$ agarose gel electrophoresis, visualized with ethidium bromide, and quantified using ImageJ software.

Western blot analysis. The rats were sacrificed after the behavioral test by deep anesthesia. The right hippocampus of rats was rapidly dissected out, frozen and stored in a deep freezer at $-80^{\circ} \mathrm{C}$ until the assays. Hippocampal tissue was homogenized in a solubilizing solution $[20 \mathrm{mM}$ Tris- $\mathrm{HCl}$ (pH 7.0), $25 \mathrm{mM} \beta$-glycerophosphate, 2 mM EGTA, $1 \%$ Triton X-100, $1 \mathrm{mM}$ vanadate, $1 \%$ aprotinin, $1 \mathrm{mM}$ phenylmethylsulfonyl fluoride, $2 \mathrm{mM}$ dithiothreitol] on ice for $40 \mathrm{~min}$. The lysate was centrifuged at $15,000 \mathrm{rpm}$ for $15 \mathrm{~min}$. The supernatant was denatured at $95^{\circ} \mathrm{C}$ for $5 \mathrm{~min}$, then by 10 or $15 \%$ SDS-polyacrylamide gel electrophoresis and transferred to a PVDF membrance. Immunostaining was performed with anti-caspase-3 antibody, anti-MAO-A antibody, anti-Hsp70 antibody and $\beta$-actin antibody. Immunoreactivity was detected with peroxidase-conjugated secondary antibody in conjunction with chemiluminesence-based film autoradiography. For quantification, ImageJ software was used.

Statistical analysis. All values are presented as means \pm SD. Data were analyzed by ANOVA followed by a Tukey-Kramer test as the post hoc test. Differences were considered statistically significant at a level of $\mathrm{P}<0.05$.

\section{Results}

Effects of GGA on the locomotor activity in the open-field test. Three weeks of CMS led to a significant decrease in locomotor activity compared with the control group, demonstrated by a decreased number of crossings and rearings (Fig. 1). By 
A

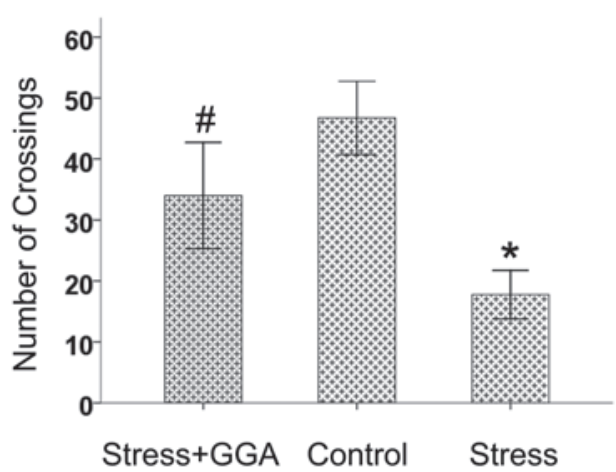

B

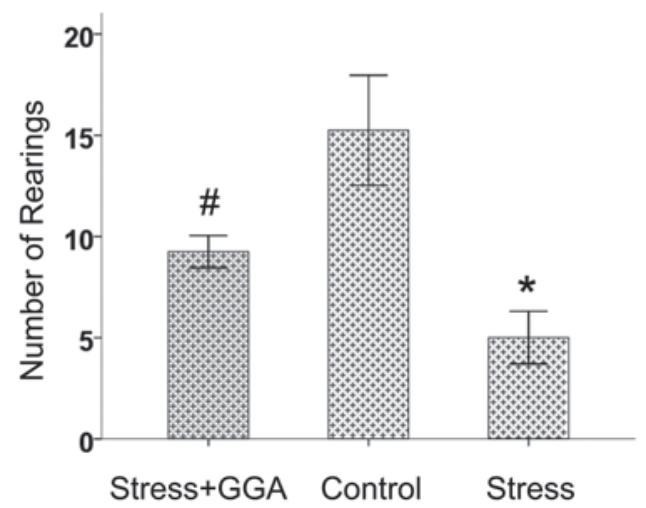

Figure 1. Effects of GGA on locomotor activity of rats after CMS. Animals were exposed to CMS and treated with GGA. (A) Number of crossings and (B) rearings were measured during the $5 \mathrm{~min}$ session. Values given are the means $\pm \mathrm{SD}(\mathrm{n}=12) .{ }^{*} \mathrm{P}<0.05$ as compared with the control; ${ }^{\prime \prime} \mathrm{P}<0.05$ as compared with the stress group. CMS, chronic mild stress; GGA, geranylgeranylacetone.

repeated treatment with GGA $(1,000 \mathrm{mg} / \mathrm{kg})$, changes in locomotor activity were almost completely reversed.

Effects of GGA on MAO-A expression in the hippocampus. It has been reported that there is an increased level of MAO-A in depression (23). RT-PCR and western blot analysis demonstrated that MAO-A mRNA and protein levels in the hippocampus were increased by CMS (Fig. 2). GGA treatment reversed these alterations, producing a significant decrease in MAO-A mRNA and protein levels in the hippocampus.

Effects of GGA on caspase-3 expression in the hippocampus. Neuronal apoptosis is involved in the pathogenesis of depression (24). Caspase-3 is a common downstream effector in the apoptosis cascade. Compared with the increased levels observed in the stress group, chronic GGA treatment resulted in a significantly decreased level of hippocampal caspase-3 mRNA and protein (Fig. 3). These results indicate that the antidepressant effect of GGA might be partly due to the suppression of neuronal apoptosis caused by CMS.

Effects of GGA on Hsp70 expression in the hippocampus. To investigate the role of Hsp70 in the antidepressant effect of GGA, we examined the Hsp70 level in the hippocampus after an open-field test by RT-PCR, western blot analysis and immunohistochemical analysis. GGA treatment significantly increased the levels of hippocampal Hsp70 mRNA and protein
A

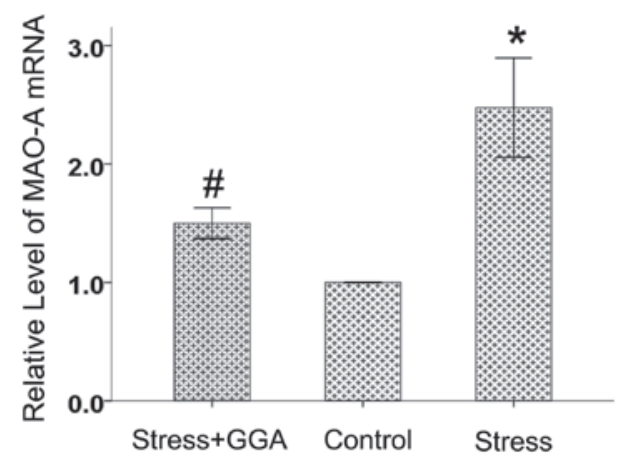

B
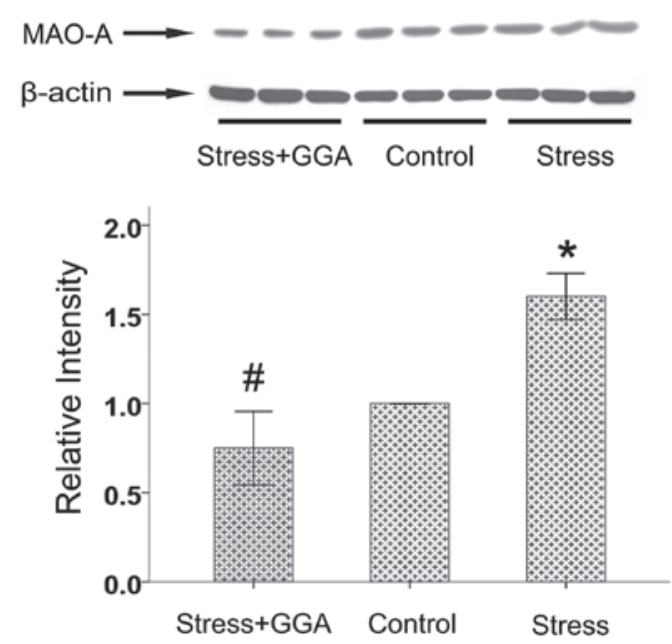

Figure 2. GGA suppressed CMS-induced increase in MAO-A level. After the open-field test, brains of rats $(n=3)$ were dissected out. (A) The level of MAO-A mRNA in the hippocampus was detected by RT-PCR. (B) The level of MAO-A protein in the hippocampus was detected by western blot analysis, and quantification is shown in graphically. ${ }^{*} \mathrm{P}<0.05$ as compared with the control; ${ }^{\text {}} \mathrm{P}<0.05$ as compared with the stress group. GGA, geranylgeranylacetone; CMS, chronic mild stress; MAO-A, monoamine oxidase-A.

(Figs. 4 and 5). These data suggest that GGA induces Hsp70 expression, which may be involved in the antidepressant effect of GGA.

\section{Discussion}

The current study demonstrates that GGA, an Hsp70 inducer, possesses an antidepressant effect. CMS in rats caused a reduction in locomotor activity and an increase in levels of monoamine oxidase-A and caspase- 3 in hippocampus. GGA treatment $(1,000 \mathrm{mg} / \mathrm{kg})$ reversed stress-induced alteration in locomotor activity and levels of MAO-A and caspase-3. Previous animal studies hava reported that $\mathrm{LD}_{50}$ values of GGA is $15,000 \mathrm{mg} / \mathrm{kg}$ in oral dose and approximately $4,000 \mathrm{mg} / \mathrm{kg}$ in intraperitoneal injection in rats and mice (21). As such, although the dose used in the present study is high, it is not acutely toxic. Therefore, GGA may be a safe and potent agent for treating depression.

It has been widely reported that depression is a consequence of diminished neurotransmission due to a decrease in neurotransmitter concentrations $(25,26)$. Among various neurotransmitters, monoamines (including noradrenaline, dopamine and serotonin) play crucial roles in the pathology of depression (27). Levels of monoamines are generally low 
A

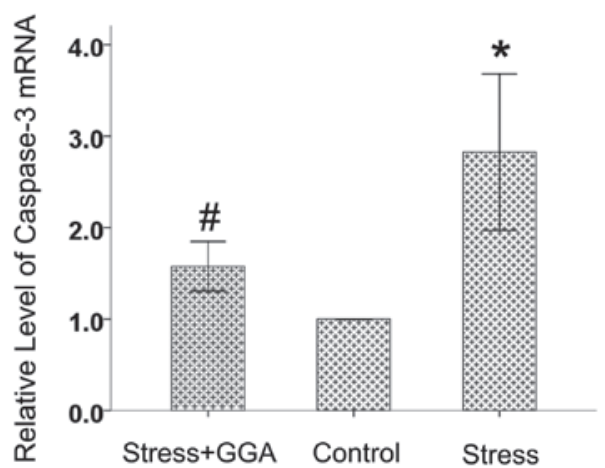

B
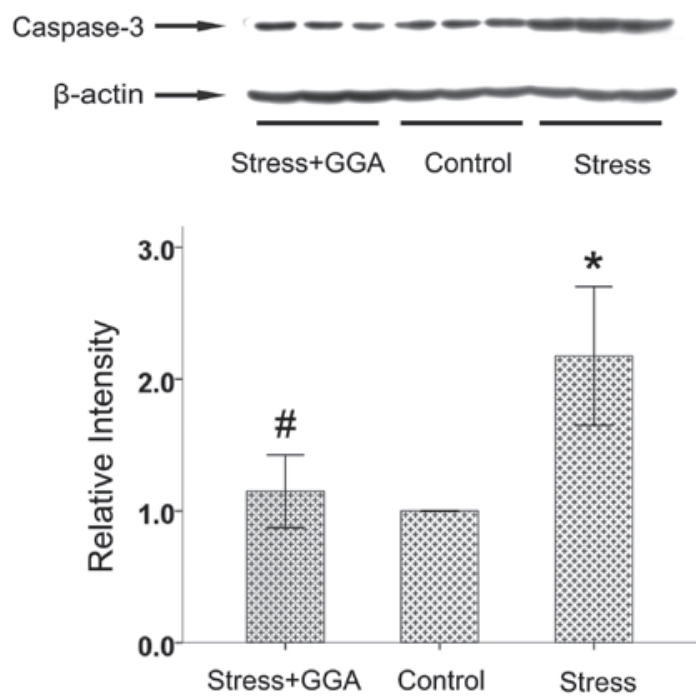

Figure 3. GGA suppressed CMS-induced increase in caspase-3 level. After the open-field test, brains of rats $(n=3)$ were dissected out. (A) The level of caspase- 3 mRNA in the hippocampus was detected by RT-PCR. (B) The level of caspase-3 protein in the hippocampus was detected by western blot analysis, and quantification is shown graphically. ${ }^{*} \mathrm{P}<0.05$ as compared with the control; " $\mathrm{P}<0.05$ as compared with the stress group. GGA, geranylgeranylacetone; CMS, chronic mild stress.
A

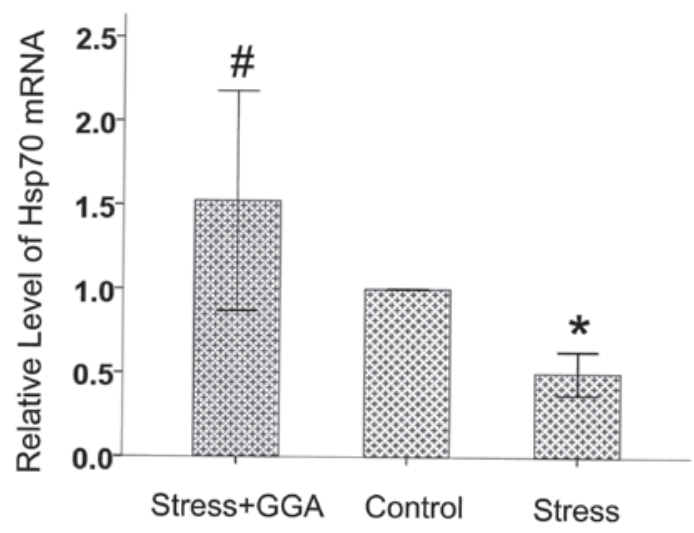

B
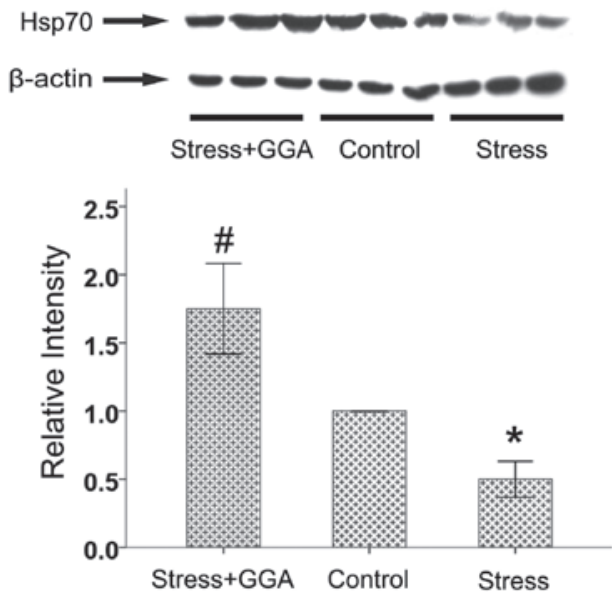

Figure 4. GGA increased Hsp70 expression. After the open-field test, brains of rats $(n=3)$ were dissected out. (A) The level of Hsp70 mRNA in the hippocampus was detected by RT-PCR. (B) The level of Hsp70 protein in the hippocampus was detected by western blot analysis, and quantification is shown graphically. ${ }^{~} \mathrm{P}<0.05$ as compared with the control; ${ }^{~} \mathrm{P}<0.05$ as compared with the stress group. GGA, geranylgeranylacetone; Hsp70, heat shock protein 70

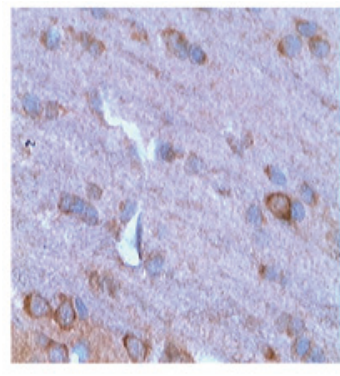

Stress+GGA

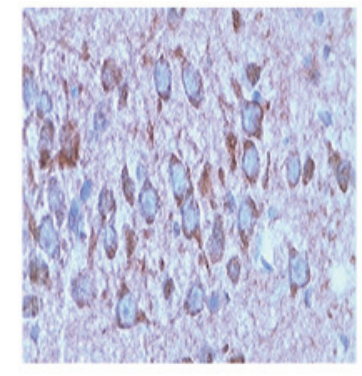

Control

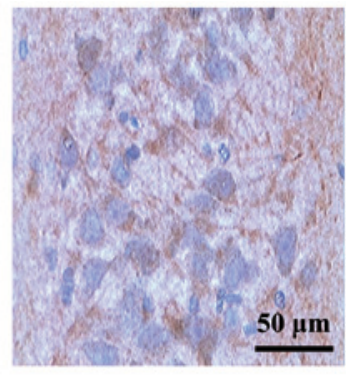

Stress

Figure 5. Representative photomicrographs of Hsp70 immunohistochemical staining in the hippocampus. After the open-field test, brains of rats (n=3) were dissected out. The level of Hsp70 protein in the hippocampus was detected by immunohistochemical analysis. Positive cells are represented as brown spots. Scale bar, $200 \mu \mathrm{m}$.

in patients with depression (23). Monoamine oxidase (MAO), the most significant enzyme that catabolizes monoamines, is a trait-dependent indicator of vulnerability to depression (28). There are two forms of MAO: MAO-A and MAO-B. MAO-A activity is believed to be associated with depression, while
MAO-B activity is believed to be associated with neurodegenerative diseases such as Parkinson's disease $(29,30)$. An elevated level of MAO-A is considered as the primary monoamine-lowering process in depression (23). In accordance with these reports, the present study observed that 
the levels of MAO-A mRNA and protein were increased in the hippocampus of CMS rats. In the present study, we also observed that GGA possessed an antidepressant effect and that the increased level of MAO-A caused by CMS was suppressed by GGA. These results suggest that the inhibitory effect of GGA on MAO-A expression may be a mechanism underlying antidepressant effects of GGA. However, further study is necessary to determine how GGA suppresses MAO-A expression. It has been reported that GGA increases thioredoxin-1, Hsp70 and prostaglandin expression in various cells, and exerts cytoprotection $(15,20,31,32)$. In the present study, we observed that GGA induced Hsp70 expression in the hippocampus. However, further studies are required to investigate whether the inhibitory effect of GGA on MAO-A expression is due to the induction of Hsp70 and/or other inducing proteins.

Although the monoamine hypothesis provides a satisfactory explanation of the mechanism underlying the pathology of depression, alternative mechanisms are also involved in the pathology of depression. Recent reports have shown an association between depression, atrophy and cell loss in the brain (33-35). Loss of neuronal and glial density is observed in post-mortem brains of patients with depression (36-39). Chronic unpredictable stress leads to neuronal apoptosis in the cerebral cortex (40). The hippocampus is particularly sensitive to stress. It has been reported that there is a selective loss of hippocampal volume in depression $(41,42)$. Loss of neurons is also observed in animal models of depression (41). It has been proposed that apoptosis is a contributing factor to the decrease in hippocampal volume and cell loss (43). Caspase-3 plays a crucial role in the execution of apoptosis (44). In the present study, we demonstrated that caspase-3 expression was increased in the hippocampus after CMS, suggesting the induction of apoptosis. Caspase- 3 could be activated in the apoptotic cell by extrinsic (death ligand) and intrinsic (mitochondrial or endoplasmic reticulum) pathways (45-48). Further studies are required to determine which apoptosis pathway is involved in the CMS-induced apoptosis. A number of studies have shown that GGA protects against various stress injuries, including acoustic injury, ischemia, age-related hearing loss and 3-nitropropionic acid-induced cochlear damage, which involves Hsp70 induction $(21,49,50)$. GGA is a lipid-soluble reagent that easily crosses the blood-brain barrier $(50,51)$. In the present study, we observed that GGA had antidepressant effects. In addition, GGA induced Hsp70 expression in the hippocampus. The activation of caspase- 3 caused by CMS was suppressed by GGA treatment. Hsp70 has been shown to inhibit the immediate apoptosis of cells exposed to numerous stresses in numerous tissues (52). The induction of Hsp70 expression by GGA may be associated with the inhibition of reactive oxygen species generation, toxicity of excitatory amino acid and apoptosis $(8,53,54)$. Accordingly, the protective effect of GGA against the apoptosis cascade by inducing Hsp70 expression may be a mechanism underlying the antidepressant effects of GGA.

In conclusion, GGA exerted an antidepressant effect in the CMS model of depression in rats and this effect may be mediated by inducing Hsp70 expression to suppress MAO-A expression and the apoptosis cascade. In addition, these results suggest that attempts to develop Hsp70 inducers are beneficial for protection against depression.

\section{Acknowledgements}

This study was supported by the Science and Technology Joint Special Fund of Yunnan Province (no. 2010CD197).

\section{References}

1. Kessler RC, Berglund P, Demler O, et al: The epidemiology of major depressive disorder: results from the National Comorbidity Survey Replication (NCS-R). JAMA 289: 3095-3105, 2003.

2. Nemeroff CB: The burden of severe depression: a review of diagnostic challenges and treatment alternatives. J Psychiatr Res 41: 189-206, 2007.

3. Rosen RC and Marin H: Prevalence of antidepressant-associated erectile dysfunction. J Clin Psychiatry 64 (Suppl 10): 5-10, 2003.

4. Evans CG, Chang L and Gestwicki JE: Heat shock protein 70 (hsp70) as an emerging drug target. J Med Chem 53: 4585-4602, 2010.

5. Parsell DA and Lindquist S: The function of heat-shock proteins in stress tolerance: degradation and reactivation of damaged proteins. Annu Rev Genet 27: 437-496, 1993.

6. Sharp FR, Massa SM and Swanson RA: Heat-shock protein protection. Trends Neurosci 22: 97-99, 1999.

7. Ooie T, Takahashi N, Saikawa T, et al: Single oral dose of geranylgeranylacetone induces heat-shock protein 72 and renders protection against ischemia/reperfusion injury in rat heart. Circulation 104: 1837-1843, 2001.

8. Guo S, Wharton W, Moseley P and Shi H: Heat shock protein 70 regulates cellular redox status by modulating glutathione-related enzyme activities. Cell Stress Chaperones 12: 245-254, 2007.

9. Kimmins S and MacRae TH: Maturation of steroid receptors: an example of functional cooperation among molecular chaperones and their associated proteins. Cell Stress Chaperones 5: 76-86, 2000.

10. Rajapandi T, Greene LE and Eisenberg E: The molecular chaperones Hsp90 and Hsc70 are both necessary and sufficient to activate hormone binding by glucocorticoid receptor. J Biol Chem 275: 22597-22604, 2000.

11. Shimizu S, Nomura K, Ujihara M, et al: An allel-specific abnormal transcript of the heat shock protein 70 gene in patients with major depression. Biochem Biophys Res Commun 219: 745-752, 1996.

12. Martini F, Fernández C, Segundo LS, Tarazona JV and Pablos MV: Assessment of potential immunotoxic effects caused by cypermethrin, fluoxetine, and thiabendazole using heat shock protein 70 and interleukin-1beta mRNA expression in the anuran Xenopus laevis. Environ Toxicol Chem 29: 2536$2543,2010$.

13. Yu J, Roh S, Lee JS, et al: The effects of venlafaxine and dexamethasone on the expression of HSP70 in rat C6 glioma cells. Psychiatry Investig 7: 43-48, 2010.

14. Allagui MS, Nciri R, Rouhaud MF, et al: Long-term exposure to low lithium concentrations stimulates proliferation, modifies stress protein expression pattern and enhances resistance to oxidative stress in SH-SY5Y cells. Neurochem Res 34: 453-462, 2009.

15. Hirakawa T, Rokutan K, Nikawa T and Kishi K: Geranylgeranylacetone induces heat shock proteins in cultured guinea pig gastric mucosal cells and rat gastric mucosa. Gastroenterology 111: 345-357, 1996.

16. Ishii Y, Kwong JM and Caprioli J: Retinal ganglion cell protection with geranylgeranylacetone, a heat shock protein inducer, in a rat glaucoma model. Invest Ophthalmol Vis Sci 44: 1982-1992, 2003.

17. Latchman DS: Heat shock proteins and cardiac protection. Cardiovasc Res 51: 637-646, 2001.

18. Yamagami K, Yamamoto $\mathrm{Y}$, Ishikawa $\mathrm{Y}$, Yonezawa K, Toyokuni S and Yamaoka Y: Effects of geranyl-geranyl-acetone administration before heat shock preconditioning for conferring tolerance against ischemia-reperfusion injury in rat livers. J Lab Clin Med 135: 465-475, 2000.

19. Katsuno M, Sang C, Adachi H, et al: Pharmacological induction of heat-shock proteins alleviates polyglutamine-mediated motor neuron disease. Proc Natl Acad Sci USA 102: 16801-16806, 2005.

20. Tanito M, Kwon YW, Kondo N, et al: Cytoprotective effects of geranylgeranylacetone against retinal photooxidative damage. J Neurosci 25: 2396-2404, 2005 . 
21. Yasuda H, Shichinohe H, Kuroda S, Ishikawa T and Iwasaki Y: Neuroprotective effect of a heat shock protein inducer, geranylgeranylacetone in permanent focal cerebral ischemia. Brain Res 1032: 176-182, 2005.

22. Luo FC, Qi L, Lv T, et al: Geranylgeranylacetone protects mice against morphine-induced hyperlocomotion, rewarding effect, and withdrawal syndrome. Free Radic Biol Med 52: 1218-1227, 2012.

23. Meyer JH, Ginovart N, Boovariwala A, et al: Elevated monoamine oxidase a levels in the brain: an explanation for the monoamine imbalance of major depression. Arch Gen Psychiatry 63: 1209-1216, 2006.

24. Duman RS: Depression: a case of neuronal life and death? Biol Psychiatry 56: 140-145, 2004.

25. Manji HK, Drevets WC and Charney DS: The cellular neurobiology of depression. Nat Med 7: 541-547, 2001

26. Mathew SJ, Manji HK and Charney DS: Novel drugs and therapeutic targets for severe mood disorders Neuropsychopharmacology 33: 2080-2092, 2008.

27. Elhwuegi AS: Central monoamines and their role in major depression. Prog Neuropsychopharmacol Biol Psychiatry 28: 435-451, 2004

28. Du L, Faludi G, Palkovits M, Sotonyi P, Bakish D and Hrdina PD: High activity-related allele of MAO-A gene associated with depressed suicide in males. Neuroreport 13: 1195-1198, 2002

29. Wouters J: Structural aspects of monoamine oxidase and its reversible inhibition. Curr Med Chem 5: 137-162, 1998.

30. Riederer P, Lachenmayer L and Laux G: Clinical applications of MAO-inhibitors. Curr Med Chem 11: 2033-2043, 2004.

31. Terano A, Shiga J, Hiraishi H, Ota S and Sugimoto T: Protective action of tetraprenylacetone against ethanol-induced damage in rat gastric mucosa. Digestion 35: 182-188, 1986.

32. Hirota K, Nakamura H, Arai T, et al: Geranylgeranylacetone enhances expression of thioredoxin and suppresses ethanolinduced cytotoxicity in cultured hepatocytes. Biochem Biophys Res Commun 275: 825-830, 2000.

33. Bremner JD: Does stress damage the brain? Biol Psychiatry 45 797-805, 1999

34. Bremner JD, Narayan M, Anderson ER, Staib LH, Miller HL and Charney DS: Hippocampal volume reduction in major depression. Am J Psychiatry 157: 115-118, 2000.

35. Colla M, Kronenberg G, Deuschle M, et al: Hippocampal volume reduction and HPA-system activity in major depression. J Psychiatr Res 41: 553-560, 2007.

36. Eastwood SL and Harrison PJ: Synaptic pathology in the anterior cingulate cortex in schizophrenia and mood disorders. A review and a western blot study of synaptophysin, GAP-43 and the complexins. Brain Res Bull 55: 569-578, 2001.

37. Lenze E, Cross D, McKeel D, Neuman RJ and Sheline YI: White matter hyperintensities and gray matter lesions in physically healthy depressed subjects. Am J Psychiatry 156: 1602-1607, 1999.
38. Nolan CL, Moore GJ, Madden R, et al: Prefrontal cortical volume in childhood-onset major depression: preliminary findings. Arch Gen Psychiatry 59: 173-179, 2002.

39. Ongür D, Drevets WC and Price JL: Glial reduction in the subgenual prefrontal cortex in mood disorders. Proc Natl Acad Sci USA 95: 13290-13295, 1998.

40. Bachis A, Cruz MI, Nosheny RL and Mocchetti I: Chronic unpredictable stress promotes neuronal apoptosis in the cerebral cortex. Neurosci Lett 442: 104-108, 2008.

41. Gould E and Tanapat P: Stress and hippocampal neurogenesis. Biol Psychiatry 46: 1472-1479, 1999.

42. McEwen BS: Physiology and neurobiology of stress and adaptation: central role of the brain. Physiol Rev 87: 873-904, 2007.

43. Lee AL, Ogle WO and Sapolsky RM: Stress and depression: possible links to neuron death in the hippocampus. Bipolar Disord 4: 117-128, 2002.

44. Woo M, Hakem R, Soengas MS, et al: Essential contribution of caspase 3/CPP32 to apoptosis and its associated nuclear changes. Genes Dev 12: 806-819, 1998.

45. Salvesen GS: Caspases: opening the boxes and interpreting the arrows. Cell Death Differ 9: 3-5, 2002.

46. Ghavami S, Hashemi M, Ande SR, et al: Apoptosis and cancer: mutations within caspase genes. J Med Genet 46: 497-510, 2009.

47. Ghribi O, Herman MM and Savory J: The endoplasmic reticulum is the main site for caspase-3 activation following aluminuminduced neurotoxicity in rabbit hippocampus. Neurosci Lett 324 217-221, 2002.

48. Shiraishi H, Okamoto H, Yoshimura A and Yoshida H: ER stressinduced apoptosis and caspase-12 activation occurs downstream of mitochondrial apoptosis involving Apaf-1. J Cell Sci 119: 3958-3966, 2006.

49. Kim YH, Song JJ, Kim YC, et al: Geranylgeranylacetone ameliorates acute cochlear damage caused by 3-nitropropionic acid. Neurotoxicology 31: 317-325, 2010.

50. Mikuriya T, Sugahara K, Sugimoto K, et al: Attenuation of progressive hearing loss in a model of age-related hearing loss by a heat shock protein inducer, geranylgeranylacetone. Brain Res 1212: 9-17, 2008.

51. Fujiki M, Kobayashi H, Abe T and Ishii K: Astroglial activation accompanies heat shock protein upregulation in rat brain following single oral dose of geranylgeranylacetone. Brain Res 991: 254-257, 2003.

52. Mosser DD, Caron AW, Bourget L, et al: The chaperone function of hsp70 is required for protection against stress-induced apoptosis. Mol Cell Biol 20: 7146-7159, 2000.

53. Yin HY, Ma XF, Liu F, Xia M and Xu AT: Protective effect of geranylgeranylacetone on cisplatin ototoxicity. Chemotherapy 55: $1-5,2009$

54. Li CY, Lee JS, Ko YG, Kim JI and Seo JS: Heat shock protein 70 inhibits apoptosis downstream of cytochrome c release and upstream of caspase-3 activation. J Biol Chem 275: 25665-25671, 2000. 